\title{
Nondiffracting images under coherent illumination
}

\author{
Boaz Salik, Joseph Rosen, and Amnon Yariv \\ Department of Electrical Engineering, California Institute of Technology, Pasadena, California 91125
}

Received May 8, 1995

\begin{abstract}
The focal depth of an arbitrary image is extended by optimization of the amplitude and phase distributions over the entire image. We propose a general method that takes advantage of a detector's (possibly nonlinear) response to incident intensity. We demonstrate the method by computer simulations and experimentally, using computer-generated holograms. (c) 1995 Optical Society of America
\end{abstract}

Since Durnin's introduction of the nondiffracting Bessel beam in 1987, ${ }^{1}$ numerous approaches have emerged for producing finite-power pseudonondiffracting (PND) beams. ${ }^{2-4}$ Recently, analytical ${ }^{5}$ and computer-optimized ${ }^{6}$ solutions for one-dimensional PND beams were discovered. The natural generalization of these developments is a method for extending the focal depth of arbitrary transverse intensity patterns, producing PND images.

For a given illumination mode, there are two general approaches to this problem: The first is the use of extended focal-depth optics (or equivalent pupil filtering) for imaging arbitrary patterns; this approach, though general, leads to a degradation of resolution and/or contrast for a given aperture size.,7 The second is the design of the phase and amplitude distributions at the image plane so that the diffracted field yields a desired transverse intensity pattern over an extended distance (focal depth). This has been attempted for continuous-phase masks ${ }^{8}$ and proven successful only for very simple transverse images. ${ }^{9,10}$ Here we introduce and demonstrate a novel method for optimizing the image-plane phase and amplitude distributions and utilizing a detector's intensity response to yield arbitrary PND binary images.

We begin by considering the image constraints imposed by the detector. A typical detector response curve $^{11}$ is shown in Fig. 1. Below some threshold intensity $I_{0}$ the incident illumination is not detected; above some intensity $I_{1}$ the illumination is detected; and between these two levels the detector behaves unpredictably. It is this intermediate region that we with to avoid.

This allowed intensity variation affords us a degree of freedom in designing the mask-instead of imposing a transmissivity of zero or one (binary transmission), we are allowed in dark regions to transmit an amplitude as high as $\sqrt{I_{0}}$ and in light regions as low as $\sqrt{I_{1}}$. Additionally, since the detector is insensitive to the field's phase, we may implement an arbitrary phase shift at each mask pixel and therefore design the phase distribution to maximize our image's focal depth.

We are now in a position to tackle the mask design problem. To extend our original depth of focus $\sigma$ by some multiple, say, to $k \sigma$, we must ensure that the image remains focused in the entire volume between the image plane and the final focused plane. Given some minimum feature size $\Delta x$, the distance $\sigma$ over which we know little diffraction will occur is ${ }^{12} \sigma=(\Delta x)^{2} / \lambda$, where $\lambda$ is the illumination wavelength. Thus, to ensure a focal depth of $k \sigma$, we must check the image at intervals of $\sigma$ (henceforth termed critical planes) and make sure that it remains focused at each of these (Fig. 2). To this end, we define an error measure (e.g., a Euclidean distance square) after thresholding that quantifies the deviation of our image from the desired one, calculate at each critical plane, and sum over all these planes to obtain the total error of our field distribution. Because we are concerned with image coordinates that may stray significantly from the $z$ axis, we use the Rayleigh-Sommerfeld formula ${ }^{13}$ to calculate the diffracted field (Fig. 3):

$$
u(x, y, z)=\iint u\left(x_{1}, y_{1}\right) \frac{z \exp (i k r)}{i \lambda r^{2}} \mathrm{~d} x_{1} \mathrm{~d} y_{1}
$$

where $\left(x_{1}, y_{1}\right)$ are the image-plane coordinates and $r^{2}=\left(x-x_{1}\right)^{2}+\left(y-y_{1}\right)^{2}+z^{2}$. Note that we assume for now quasi-monochromatic, coherent illumination and an imaging system within its resolution limit (therefore the complex mask distribution equals the image-plane field distribution). Next we independently optimize the mask's amplitude and phase distributions, a process that (as shown in a previous publication ${ }^{6}$ ) converges to an error minimum for the complex mask distribution. In this case we iteratively perform a pattern search optimization ${ }^{14}$ on the amplitude and phase until a common minimum is attained. We optimize until a postthresholding error of zero is

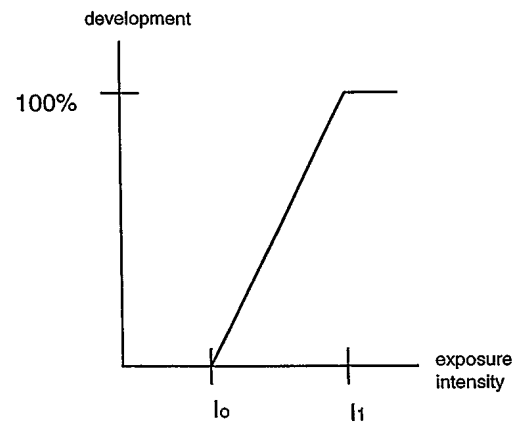

Fig. 1. Typical detector response versus exposure intensity. 


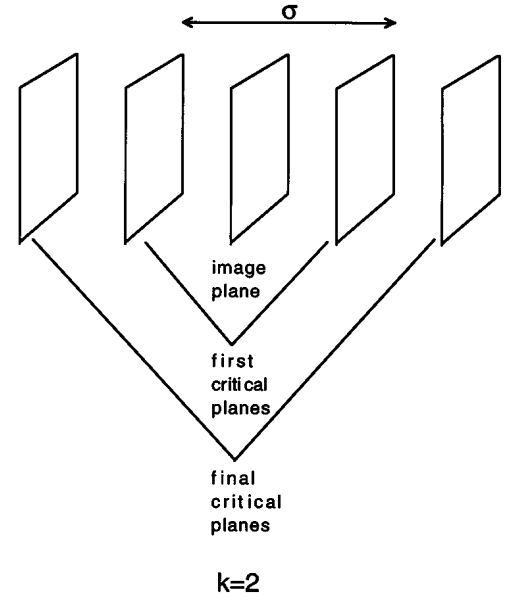

Fig. 2. Relationship of critical planes to classical depth of focus $\sigma$.

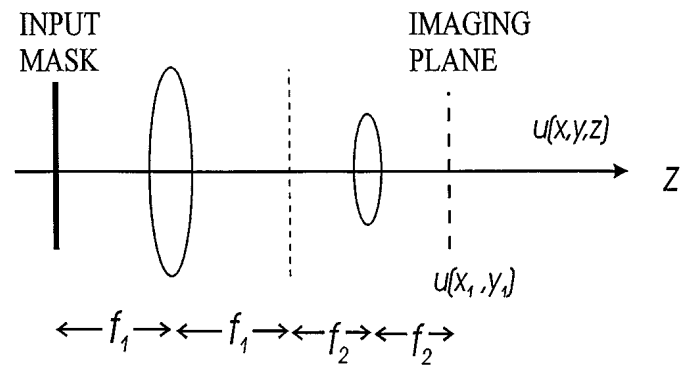

Fig. 3. Schematic diagram of a typical imaging system.

reached on all critical planes. If zero error is not attainable, we consider the problem unsolvable by our method, given mask resolution and detector thresholding parameters. At this point we have the desired complex mask distribution, which may be physically realized in several different ways. ${ }^{15}$

To demonstrate the generality of this technique, we chose the aperiodic, multicornered image shown in Fig. 4(a). The stimulated intensity patterns after diffraction for a binary transmission mask and an optimized amplitude-phase mask are shown in Fig. 4(b), and the postthresholding detector response is shown in Fig. 4(c). Although the binary-mask image becomes unacceptably distorted at the final critical plane, the optimized image remains completely undistorted (note that one pixel corresponds to the resolution limit of the imaging system, $\lambda f / D$ ).

Finally, we implemented the amplitude-phase masks as Fourier-transform holograms, as shown in Fig. 5. Given our desired mask distribution $g\left(x_{1}, y_{1}\right)$, we Fourier transform to $G\left(x_{0}, y_{0}\right)$ and then take

$$
\begin{aligned}
G^{\prime}\left(x_{0}, y_{0}\right)= & \min \left(\operatorname{Re}\left\{G\left(x_{0}, y_{0}\right) \exp \left[i 2 \pi u\left(x_{0}+y_{0}\right)\right]\right\}\right) \\
& +\operatorname{Re}\left\{G\left(x_{0}, y_{0}\right) \exp \left[i 2 \pi u\left(x_{0}+y_{0}\right)\right]\right\},
\end{aligned}
$$

yielding a real-positive mask. Multiplying $G$ by $\exp \left[i 2 \pi u\left(x_{0}+y_{0}\right)\right]$ shifts our pattern to a propagation angle $\theta=\lambda u$ in both the $x z$ and $y z$ planes; taking the real part (or, equivalently, adding the complex conjugate) causes the inverted pattern to appear at an angle $-\theta$. Finally, adding the constant simply introduces a focused spot that does not interfere with our pattern if $\theta$ is large enough.

Using a CCD to record the transverse images and postprocessing to simulate detector thresholding, we

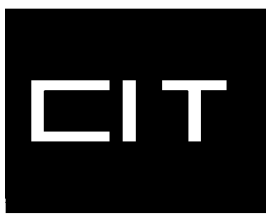

(a)

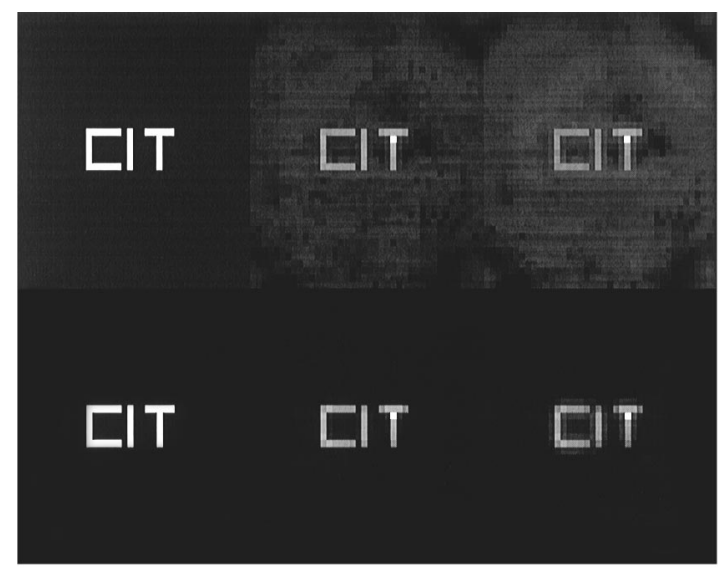

(b)

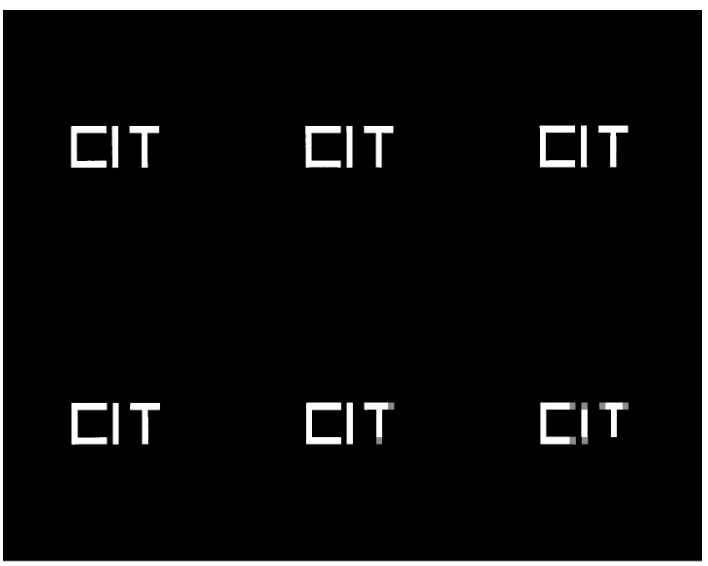

(c)

Fig. 4. (a) Desired postthresholding image. (b) Simulated intensity pattern before thresholding at the image plane $(z=0)$ and the two critical planes $z=5_{\text {pixels }}$ and $z=$ $10_{\text {pixels. }}$ (c) Simulated intensity pattern after thresholding at the image plane $(z=0)$ and the two critical planes $z=5_{\text {pixels }}$ and $z=10_{\text {pixels. }}$. Here one longitudinal pixel $=(\Delta x)^{2} / 5 \lambda=\sigma / 5$, or one fifth of the normal depth of focus.

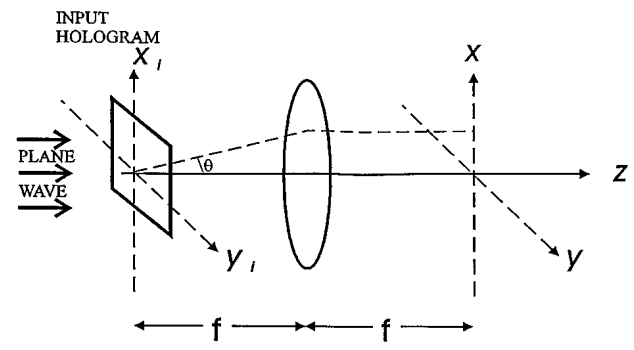

Fig. 5. Experimental configuration for realizing the mask function $g(x, y)$. 


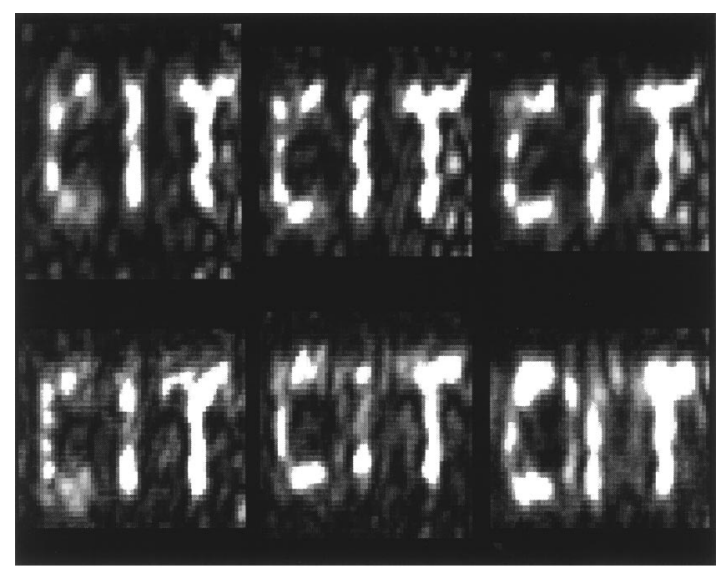

(a)

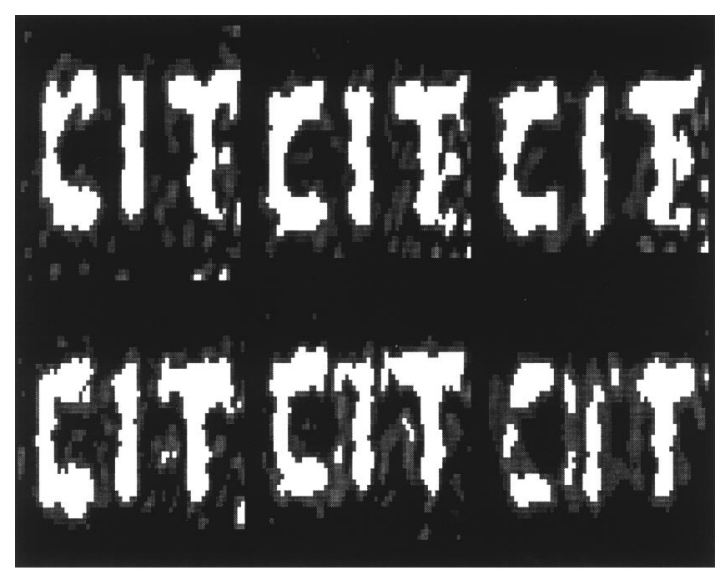

(b)

Fig. 6. (a) Measured intensity pattern before thresholding at the image plane $(z=0)$ and the critical planes $z=0.7 \mathrm{~cm}$ and $z=1.4 \mathrm{~cm}$. (b) Measured intensity pattern after thresholding at the image plane $(z=0)$ and the critical planes $z=0.7 \mathrm{~cm}$ and $z=1.4 \mathrm{~cm}$. Images were produced using setup of Fig. 5, with $f=100 \mathrm{~cm}, \theta=2.5 \mathrm{mrad}$, and illumination $\lambda=633 \mathrm{~nm}$.

obtained the results in Figs. 6(a) and 6(b), respectively. As predicted by our simulations, the optimized mask images remain focused significantly farther than the binary images.

In this example, we improved focal depth approximately twofold by using optimized amplitude-phase masks; in practice, the improvement in focal depth depends on the sharpness of the detector's threshold, attainable mask resolution, and pattern irregularity. Of these, detector thresholding is by far the most influential parameter, since a sharper threshold means larger tolerable amplitude fluctuations and more freedom in optimizing the mask. Because the assumed thresholding intensity response applies, with appropriate parameters, to a wide range of detectors and object media, from rod cells in the human eye $\mathrm{e}^{16}$ to avalanche photodiodes ${ }^{9}$ to photolithographic photoresist, ${ }^{17}$ this method is quite general, but its success depends intimately on the application for which it is used.

This research is based on work supported under a National Science Foundation Graduate Research Fellowship and grants from the Advanced Research Projects Agency and the U.S. Air Force Office of Scientific Research.

\section{References}

1. J. Durnin, J. Opt. Soc. Am. A 4, 651 (1987).

2. R. H. Jordan and D. G. Hall, Opt. Lett. 19, 427 (1994).

3. J. Rosen, Opt. Lett. 19, 369 (1994).

4. J. Rosen, B. Salik, and A. Yariv, "Pseudonondiffracting beam generated by radial harmonic function," submitted to J. Opt. Soc. Am. A.

5. J. Rosen, B. Salik, A. Yariv, and H. K. Liu, Opt. Lett. 20, 423 (1995).

6. B. Salik, J. Rosen, and A. Yariv, J. Opt. Soc. Am. A 12, 1702 (1995).

7. J. Ojeda-Castaneda and L. R. Berriel-Valdos, Appl. Opt. 29, 994 (1990).

8. R. Piestun and J. Shamir, Opt. Lett. 19, 771 (1994).

9. Y. Liu, A. K. Pfau, and A. Zakhor, Proc. Soc. Photo-Opt. Instrum. Eng. 1674, 14 (1992).

10. Y. C. Pati and T. Kailath, J. Opt. Soc. Am. A 11, 2438 (1994).

11. B. E. A. Saleh and M. C. Teich, Fundamentals of Photonics (Wiley, New York, 1991), pp. 665-681.

12. M. D. Levenson, Jpn. J. Appl. Phys. 33, 6765 (1994).

13. J. W. Goodman, Introduction to Fourier Optics (McGraw-Hill, New York, 1968), p. 45.

14. L. R. Foulds, Optimization Techniques (SpringerVerlag, New York, 1981), pp. 329-335.

15. R. A. Ferguson, Proc. Soc. Photo-Opt. Instrum. Eng. 2197, 130 (1994).

16. A. Knowles and H. J. A. Dartnall, in The Eye IIB, H. Davidson, ed. (Academic, Orlando, Fla., 1977), pp. $461-469$.

17. D. J. Elliott, Integrated Circuit Fabrication Technology (McGraw-Hill, New York, 1989), p. 232. 